\title{
DETERMINANTES DE LAS METAS DE EDUCACIÓN Y LOS OBJETIVOS DE DESARROLLO DEL MILENIO EN HONDURAS
}

\author{
(1) María Auxiliadora López Méndez, \\ Universidad Nacional Autónoma de Honduras (UNAH) \\ Facultad de Ciencias Económicas, Administrativas y Contables (FCEAC) \\ Instituto de Investigaciones Económicas y Sociales (IIES) \\ Ciudad universitaria, edificio C2, primer piso, Tel/fax: + (504) 2216-6100 Ext. 100894 \\ maria.lopez@unah.edu.hn \\ Marcelo T. LaFleur \\ Department of Economic and Social Affairs United Nations, \\ New York, USA \\ lafleurm@un.org ${ }^{1}$ \\ DOI http://dx.doi.org/10.5377/eya.v8i2.5615
}

\section{RESUMEN}

Durante el periodo 1990-2012, la educación primaria ha sido una prioridad en la política educativa de Honduras, con importancia similar en los Objetivos de Desarrollo del Milenio. El objetivo del presente estudio es identificar y cuantificar los factores determinantes de la matrícula escolar, la promoción de grado, y las tasas de graduación en el nivel primario, y con ello llegar a una comprensión de la función de los diferentes factores en la terminación oportuna de la educación primaria por la juventud de Honduras. Este articulo tiene como objetivo primordial cuantificar la relación entre resultados de la educación y las variables socioeconómicas y políticas para el desarrollo del modelo de equilibrio general MAMS de Honduras, este insumo también permitirá identificar las políticas que tienen más probabilidades de mejorar los resultados y alcanzar los objetivos de desarrollo.

Se encontró que las variables socioeconómicas y políticas varían en su impacto en los resultados educativos. Y se llegó a la conclusión de que existen oportunidades significativas para los responsables hacedores de política pública, para aumentar el impacto de las políticas en los resultados educativos, en particular la eficacia del gasto en los logros de la educación oportuna, y tomando en consideración las mejoras en las condiciones socioeconómicas. Una lección clave de este estudio es que proporciona un ejemplo riguroso de cómo analizar el efecto de la política en los resultados, lo que requiere datos detallados y técnica estadística adecuada, para informar mejor al momento de tomar decisiones de política pública.

Palabras clave: Modelo de construcción y estimación, Análisis microeconómico del Desarrollo Económico, Gasto de Gobierno y Educación, Análisis de la Educación. 


\title{
DETERMINANTS OF EDUCATION GOALS AND THE MILLENNIUM DEVELOPMENT GOALS IN HONDURAS
}

\author{
(1) María Auxiliadora López Méndez, \\ Universidad Nacional Autónoma de Honduras (UNAH) \\ Facultad de Ciencias Económicas, Administrativas y Contables (FCEAC) \\ Instituto de Investigaciones Económicas y Sociales (IIES) \\ Ciudad universitaria, edificio C2, primer piso, Tel/fax: + (504) 2239-1849 \\ maria.lopez@unah.edu.hn \\ Marcelo T. LaFleur \\ Department of Economic and Social Affairs United Nations, \\ New York, USA \\ lafleurm@un.org² \\ DOI http://dx.doi.org/10.5377/eya.v8i2.5615
}

\begin{abstract}
Since 1990-2012, basic primary education has been a priority in Honduras' education policy, with similar importance in the Millennium Development Goals. The objective of this study is to identify and quantify the determinants of school enrollment, grade promotion, and graduation rates in primary school, and to reach an understanding of the role of various factors in the timely completion of primary education by Honduras' youth. This work aims primarily to quantify the relationship between education outcomes and socio-economic and policy variables to inform the MAMS general equilibrium model of Honduras, but it also serves to identify the policies that are most likely to improve outcomes and reach development objectives. We find that socioeconomic and policy variables vary in their impact in education outcomes. We conclude that there are significant opportunities for policy makers to increase the impact of policies on education outcomes, particularly the effectiveness of spending on timely education achievement, but also though improvements in socio-economic conditions. A key lesson of this study is that it provides a rigorous example of how to analyze the effect of policy on outcomes, requiring detailed data and proper statistical technique to provide better information when making policy decisions.
\end{abstract}

Keywords: Model Construction and Estimation, Microeconomic Analyses of Economic Development, Government Expenditures and Education, Analysis of Education.

1 Corresponding author

Revista Economía y Administración (E\&A) / VOL. 8 


\section{INTRODUCCIÓN}

Dificultades económicas y sociales de orden global, como la reciente crisis financiera internacional o fenómenos de naturaleza interna y externa, provocan incertidumbre al momento de diseñar políticas de desarrollo humano. Antes de la crisis, estudios de países de América Latina y el Caribe demostraban que sería posible alcanzar los Objetivos y Metas de Desarrollo del Milenio (ODM) en 2015, con la necesidad de inversiones y otras intervenciones públicas adicionales (Vos, Sánchez, and Kaldewei 2008). La evidencia apunta a una necesidad de estrategias de financiamiento de este costo adicional bien consideradas debido a los efectos macroeconómicos que tal financiamiento se esperaba tener. La experiencia demuestra que una política de desarrollo que logre alcanzar los ODMs va acompañada de un aumento sostenido en el gasto social, un crecimiento en la demanda laboral, una mejora en la distribución del ingreso, y una estrategia de financiamiento que incluya mayores recaudaciones de impuestos junto con financiamiento externo.

Otro estudio estimó el costo necesario para alcanzar las metas de desarrollo en los 18 países de America Latina y el Caribe analizados. En el caso de las metas de educación primária, mortalidad materna e infantil, y acceso a servicios de agua y saneamiento, los gastos adicionales deberian de ser entre el 1\% y 7\% del PIB cada año en comparación con el escenario base estudiado. El país con la mayor necesidad de incrementar sus gastos es justamente Honduras (Rob Vos et al. 2010). Los costos adicionales para alcanzar los ODMs figuraban ser razonables para la región, desde que las estrategias de financiamiento del gasto adicional sean consideradas. Los impactos globales de fines de 2008 y 2009, además de los problemas economicos que el mundo todavia enfrenta, cambiaran la perspectiva.

Un analisis del impacto de la crisis global sobre la posibilidad de alcanzar los ODM en seis países de América Latina encuentra que los hubo gran incidencia. En los países de bajo ingreso, el costo adicional para alcanzar las metas seria de entre $1.6 \%$ y $3.4 \%$ del PIB por año entre 2010 y 2015 , comparado con los costos necesarios para alcanzar las metas estimadas antes de la crisis. Otra vez Honduras tendria la mayor necesidad de aumentar sus gastos en educación, salud, y servicios básicos (3,4\% del PIB por año), por encima de los $7 \%$ adicionales estimados antes de la crisis (Sánchez y Vos 2010).

El costo total esconde los detalles de distintas politicas de intervención y de financiamiento. Para tener bien claro cuales son las opciónes más eficientes para alcanzar los objetivos de desarrollo de un país, es necesario simular las propuestas, su financiamiento, y su impacto. Gobiernos de diversos países han expresado la necesidad de fortalecer las capacidades técnicas del gobierno para analizar sus estrategias de desarrollo. En respuesta a esta demanda de apoyo técnico, la División de Análisis y Políticas del Desarrollo del Departamento de Asuntos Económicos y Sociales de las Naciones Unidas (DPAD/UN-DESA, por sus siglas en inglés) - por medio del Programa de las Naciones Unidas para el Desarrollo (PNUD) representado en cada país - lleva a cabo el proyecto de creación de capacidades "Fortalecimiento de la Coherencia entre las Políticas Macroeconómicas y Sociales mediante un Modelado Macro-Micro Integrado".

Por medio de esta iniciativa se busca hacer una transferencia estructurada de herramientas de uso amigable que sirvan de apoyo a técnicos en el proceso de:

- Evaluar los impactos de eventos externos en el desarrollo, principalmente en el cumplimiento de los Objetivos de Desarrollo del Milenio (ODM).

- Formular estrategias que permitan aliviar o compensar en su totalidad los impactos adversos de esos eventos.

- Estar mejor preparados para anticipar y hacerle frente a episodios de crisis, a fin de evitar retrocesos en el desarrollo humano.

DPAD/UN-DESA realizó el trabajo de adaptación del 
modelo de equilibrio general MAMS (Maquette for MDG Simulation, en inglés) al contexto hondureño, el cual requirió, entre otras, una estimación de las elasticidades de los cuatro ODM, que forman parte del módulo de desarrollo humano de MAMS, en relación con ciertos determinantes específicos. Por este motivo, la especificación de los modelos econométricos en esta nota sigue la especificación del modelo de equilibrio general MAMS.

El presente estudio se concentra en estimar las elasticidades para los determinantes de los logros educativos (ODM 2A), describiendo la metodología y los pasos necesarios para estimar el efecto de varios factores socioeconómicos sobre tres objetivos educativos: la matricula, la tasa de promoción, y la graduación y continuación al ciclo educativo siguiente. Otras notas técnicas presentan las estimaciones de los determinantes de la salud materna e infantil (ODM 4A y 5A), $\mathrm{y}$ del acceso a los servicios básicos de agua y saneamiento (ODM 7C). Los archivos necesarios para replicar los resultados están disponibles al contactar los autores.

TABLA 1. Descripción de los ODM en MAMS

\begin{tabular}{|c|c|c|}
\hline ODM & Definición & Instrumento en MAMS \\
\hline ODM 2A & $\begin{array}{l}\text { Lograr la enseñanza primaria universal y } \\
\text { su meta es asegurar que para el año } 2015 \text {, } \\
\text { los niños y las niñas terminen un ciclo } \\
\text { completo de enseñanza primaria. }\end{array}$ & $\begin{array}{l}\text { La culminación a tiempo de la educación } \\
\text { en cada ciclo, lo cual incluye } 3 \text { aspectos: la } \\
\text { matricula, la promoción, y la continuación } \\
\text { al siguiente ciclo educativo }\end{array}$ \\
\hline ODM 4A & $\begin{array}{l}\text { Reducir la mortalidad de los niños menores } \\
\text { de } 5 \text { años. Tiene como meta reducir en } \\
\text { dos terceras partes, entre } 1990 \text { y } 2015 \text {, la } \\
\text { mortalidad de los niños menores de } 5 \text { años. }\end{array}$ & La tasa de mortalidad infantil o en la niñez. \\
\hline ODM 5A & $\begin{array}{l}\text { Mejorar la salud materna y una meta es } \\
\text { reducir, entre } 1990 \text { y } 2015 \text {, la mortalidad } \\
\text { materna en tres cuartas partes. }\end{array}$ & La tasa de mortalidad materna. \\
\hline ODM 7C & $\begin{array}{l}\text { Garantizar la sostenibilidad del medio } \\
\text { ambiente y una de las mestas es reducir a } \\
\text { la mitad, para el año } 2015 \text {, el porcentaje } \\
\text { de personas sin acceso sostenible al } \\
\text { agua potable y a servicios básicos de } \\
\text { saneamiento. }\end{array}$ & $\begin{array}{l}\text { Acceso a fuentes de agua y saneamiento } \\
\text { mejorados. }\end{array}$ \\
\hline
\end{tabular}

Fuente: Elaboración propia con datos de ODM-Honduras.

\section{ESTADÍSTICAS DESCRIPTIVAS DEL SECTOR DE EDUCACIÓN EN HONDURAS}

Según estadísticas de cobertura, gasto por alumno y densidad de alumnos por maestro, la educación pública se concentra en el sector primario, mientras que su participación es mucho menos relevante en el sector secundario y solo vuelve a crecer en el sector terciario. Cerca del $80 \%$ de los niños estudian en escuelas públicas en el nivel primario, mientras que en el nivel secundario ese porcentaje disminuye significativamente y solo llega al $20 \%$.

Son notorios los avances significativos en el aumento de las tasas de matrícula en la educación primaria, los indicadores de eficiencia se mantienen en niveles muy bajos. Es contra intuitivo en vista de que Honduras se destaca por asignar un alto porcentaje de su PIB al gasto para la educación. 
Figura No.1. Gasto en Educación como porcentaje del PIB

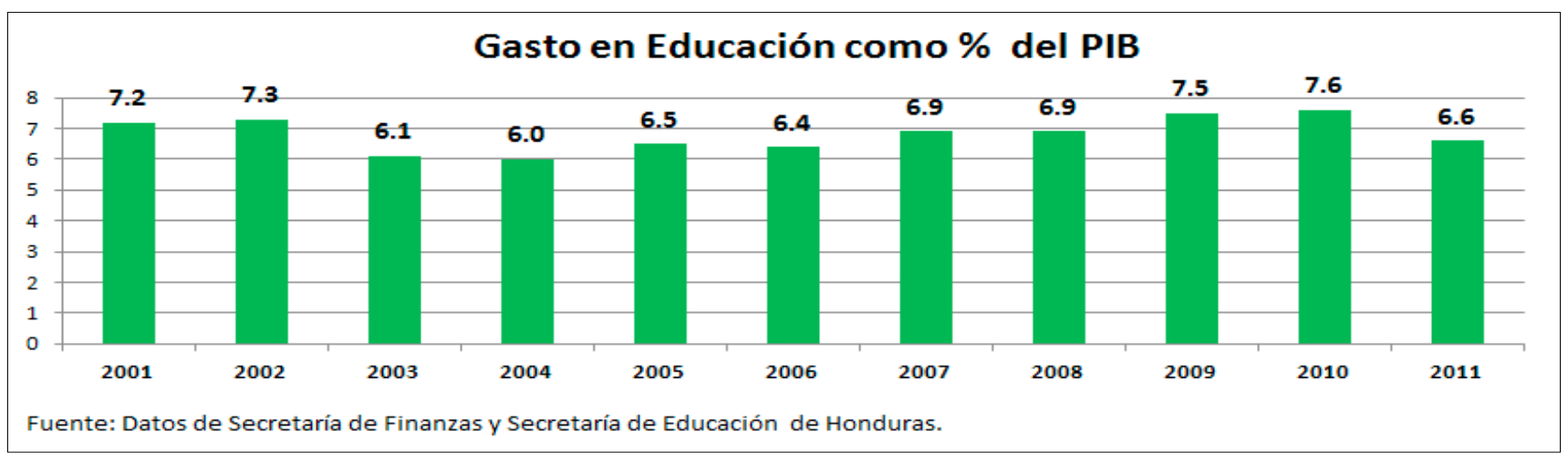

Fuente: Elaboración propia con datos de la Secretaria de Finanzas.

Como se indicó en el párrafo anterior, los indicadores de resultado no son proporcionales a los altos niveles de gasto en el sector, como se puede observar en la figura No. 1, las tasas de repitencia no reportan la tendencia esperada en vista que para 2011 en ambos dominios la tasa supera el $8 \%$ en respuesta a este indicador la tasa de matrícula neta para el mismo año desciende a $88.6 \%$ a nivel rural y a $91.6 \%$ a nivel urbano.

No obstante, las tasas de asistencia revelan que la educación primaria ha alcanzado. Niveles satisfactorios, sin embargo, para el nivel prebásico y secundario surgen deficiencias en el sentido de estancamiento de dichos indicadores no mostrando crecimiento cómo se esperaría. Tal es el caso de que sólo un $46 \%$ de la población total en edad de asistir al nivel de educación prebásica, tiene acceso a este nivel. Por otra parte, la cobertura neta del primero y segundo ciclo de educación básica alcanzo el $86.6 \%$ de la población total. Para el tercer ciclo, la cobertura llego a un $61 \%$ de la población, y en educación media solamente se atiende a un 39\%. Esto representa un serio problema, por cuanto es en este nivel donde se forma la fuerza laboral emergente que el país necesita para su desarrollo.

Por su parte, se observa que la asistencia a nivel de diversificado reporta la misma dinámica de estancamiento de sus niveles previos de educación, en vista de que, tanto en 2010 como en 2011, las proporciones de alumnos asistiendo a este nivel se mantienen en un $28 \%$, lo cual es preocupante en vista que menos de un tercio de la población llega a este nivel educativo y por tanto es una preimagen del reducido número de alumnos cursando estudios superiores universitarios. Ampliando más el horizonte de análisis tenemos que para 2011, según datos de la EPHPM la incidencia de la pobreza afecto al $67.6 \%$ de la población que vive en hogares pobres, y más fuertemente a aquellos que viven en zonas rurales $(70 \%)$ donde el acceso a educación fue significativamente inferior dadas las condiciones de vida a las que se enfrenta dicho grupo, en comparación a las personas de hogares pobres $(64.7 \%)$ que vivieron en el área urbana.

\section{MARCO TEÓRICO}

\subsection{Contexto socioeconómico en la evolución de la educación en los objetivos de desarrollo del milenio en Honduras}

En los últimos 11 años, Honduras ha registrado un crecimiento económico promedio de $4.2 \%$ donde con comportamientos anuales muy variables. Durante el período comprendido entre 20022007, como resultado de un contexto económico internacional favorable, el crecimiento pasó de $3.8 \%$ en 2002 , hasta una tasa superior al $6 \%$ en 2007. No obstante, a partir de 2008, dado el efecto de la crisis financiera internacional, la tasa de crecimiento se reduce a $4 \%$ y al sumarse la crisis política interna en 2009 , la economía de Honduras registró una caída de $2.4 \%$ en ese año. Aunque esta caída se revierte en 2010, esta dinámica de crecimiento retraso aún más la salida 
de la mayoría de la población de la situación de pobreza. Según el Banco Central de Honduras $(\mathrm{BCH})$, en 2012, la economía hondureña creció $3.3 \%$ tasa inferior a la de $3.7 \%$ que se registró en los dos años precedentes, debido a la continuada debilidad de la demanda tanto externa como interna, en particular de la inversión.

En concordancia con la evolución del crecimiento económico, el ingreso nacional bruto per cápita ha sido muy voluble, después de registrar incrementos significativos entre 2005 y 2008 , que dieron un empuje en algunos indicadores sociales. Este crecimiento se desacelero en el 2009. Se estanco en 2012, lo que sin duda indico negativamente en el logro de los principales ODM.

El panorama fiscal actual del país no fue muy halagador al observar que el déficit del sector público combinado a partir de 2009 se mantuvo en niveles elevados, y para 2012 alcanzó un 3.8\% del PIB superando significativamente al observado el año anterior (2,5\% del PIB). El gasto corriente continuó absorbiendo la mayor parte de los recursos, y el déficit tuvo su origen en el aumento de las transferencias corrientes al resto del sector público y al sector privado para el subsidio de la educación, la energía y al transporte urbano, así como el pago de intereses de la deuda.

Las necesidades de financiamiento llevaron a un aumento de la deuda interna del gobierno central, por lo que se ha observado que la deuda pública de Honduras después de registrar disminuciones importantes entre 2006 y 2008 (US\$3,216 en 2008) por la condonación internacional, a partir de 2009 se incrementó sostenidamente, hasta alcanzar en 2012, un nivel de US\$ 6,397 millones, superior al endeudamiento que se tenía en 2005. La principal dificultad que se tuvo es que el mayor porcentaje de la nueva deuda estuvo contratada en términos no concesionales o sea comerciales lo que incide en un servicio de deuda muy elevado, lo que redujo la disponibilidad de recursos que pudieron destinarse a la ejecución de programas sociales, que indico en la reducción de la pobreza (MT LaFleur, 2014).

La ausencia de solución al problema fiscal imposibilitó concretar un nuevo acuerdo con el
Fondo Monetario Internacional durante 2012, lo que restringió el acceso a nuevos recursos para financiar el desarrollo económico. La menor disponibilidad de recursos fiscales y externos impacto en la disminución del gasto social, teniendo como resultado menor disponibilidad de inversión en infraestructura social (MT LaFleur, 2014). Esto deriva en el deterioro de los activos sociales ya existentes por la falta de mantenimiento, así como en un menor acceso de la población pobre a servicios de agua potable y saneamiento, afecta a los hogares pobres.

\subsection{Mercado laboral}

El Instituto Nacional de Estadística (INE) estimo que, de 3,243,877 de los ocupados del país. El $38.2 \%$ se empleó en la agricultura, en el comercio está el $21.9 \%$ y en la industria el $13.4 \%$. Estas tres ramas de actividad concentraron más del $70 \%$ de los ocupados. Existen una cantidad considerable de personas en condición de asalariados ya que de cada 100 ocupados 42 efectuaron sus actividades laborales a cambio de un salario, 45 de cada 100 hondureños realizaron las actividades laborales por su cuenta (no tuvieron un empleador) y finalmente el $13 \%$ restante son trabajadores no remunerados, que de alguna forma trabajaron, pero no recibieron un pago a cambio (INE, 2013).

La tasa de desempleo abierto (TDA) ${ }^{3}$ para el año 2012 alcanzó el 3.6\% de la población económicamente activa. El problema del desempleo es en su mayoría urbano, potenciado por la migración constante de personas del campo a la ciudad y la poca capacidad del mercado laboral para absorber esta fuerza de trabajo. Además del desempleo, el problema del subempleo requiere atención. Personas que trabajan menos de 36 horas a la semana y desean trabajar más forman parte del subempleo "visible". El subempleo "invisible" se define como las personas que trabajan más de 36 horas y tuvieron ingresos inferiores a un salario mínimo (MT LaFleur, 2014). En 2012, la tasa de subempleo visible e invisible reportaron el 10.5\% y el $43.6 \%$ respectivamente. (Ver figura No. 2)

\footnotetext{
3 Personas que quieren trabajar y no encuentran trabajo.
} 


\subsection{Pobreza y distribución del ingreso}

La pobreza en el país es un fenómeno que afecta en gran medida a la sociedad hondureña. En el año 2001 el $63.7 \%(786,529)$ de los hogares presentaban esta situación. Para el año 2012 el $66.5 \%(1,206,698)$ de los hogares son pobres lo que se traduce en 5,889,545 personas, teniendo mayor agudeza en el área rural que el área urbana con un $74.1 \%$ y $67.6 \%$ de los hogares respectivamente.

Otros indicadores muestran el comportamiento de la pobreza en el país son la brecha ${ }^{4}$ y severidad de la pobreza. La brecha de la pobreza refleja el total de pobreza y, por tanto, proporciona una idea de la profundidad de las carencias de ingresos o consumo que definen una situación de pobreza. En el año 2001 este indicador al nivel nacional era del 53.3\%. Para el 2012 la brecha fue del 52.8\%.

La severidad refleja la situación de los más pobres dentro de la pobreza, dando más peso a los más pobres en el cálculo. En el 2001, la severidad se estimó en $22.3 \%$ existiendo mayor diferencia en lo rural que en lo urbano con un $15.8 \%$ y $28.6 \%$ respectivamente. Para el 2012, a nivel nacional, se observó un grado de severidad de $22.9 \%$, incrementándose la desigualdad en mayor medida en el área rural que llega al $25.5 \%$ en comparación con el área urbana, cuyo porcentaje fue del $20.3 \%$.

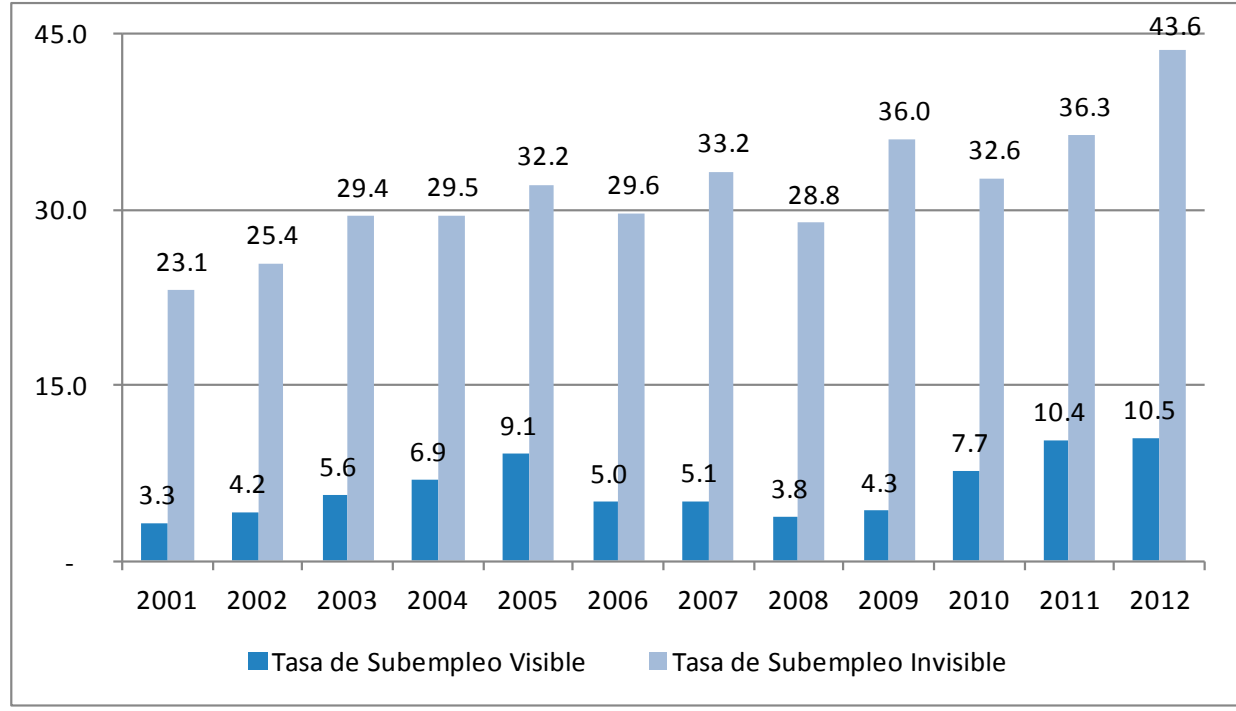

Figura No.2 Tasa de Subempleo Invisible y Tasa de Subempleo Visible

Fuente: Elaboración propia con datos de la Encuesta Permanente de Hogares de Propósitos Múltiples (EPHPM), INE, 2001-2012

\footnotetext{
4 Brecha de Pobreza, diferencia agregada entre el ingreso de las

personas (u hogares) pobres y el valor de una canasta básica de bienes

y servicio (o línea de pobreza), expresada como porcentaje de este

último valor y dividida para la población total.
} 
TABLA No.2. Proporción de la pobreza en los hogares, 2001-2012

\begin{tabular}{|c|c|c|c|c|}
\hline Período & No pobres & $\begin{array}{c}\text { Pobreza } \\
\text { Relativa }\end{array}$ & $\begin{array}{c}\text { Pobreza } \\
\text { Extrema }\end{array}$ & Pobreza Total \\
\hline 2001 & 36.3 & 19.5 & 44.2 & 63.7 \\
\hline 2002 & 35.2 & 19.2 & 45.6 & 64.8 \\
\hline 2003 & 34.7 & 18.6 & 46.7 & 65.3 \\
\hline 2004 & 35.4 & 18.4 & 46.2 & 64.6 \\
\hline 2005 & 36.3 & 17.6 & 46.0 & 63.7 \\
\hline 2006 & 40.1 & 19.6 & 40.4 & 59.9 \\
\hline 2007 & 41.8 & 20.8 & 37.5 & 58.2 \\
\hline 2008 & 40.8 & 22.9 & 36.2 & 59.2 \\
\hline 2009 & 41.2 & 22.3 & 36.4 & 58.8 \\
\hline 2010 & 40.0 & 20.9 & 39.1 & 60.0 \\
\hline 2011 & 38.1 & 20.3 & 41.6 & 61.9 \\
\hline 2012 & 30.7 & 20.5 & 46.0 & 66.5 \\
\hline
\end{tabular}

Fuente: Instituto Nacional de Estadísticas (INE). XLIII Encuesta Permanente de Hogares de Propósitos Múltiples (EPHPM).

En cuanto a la distribución de ingreso y la desigualdad en Honduras, según CEPAL (2011) la proporción de los ingresos del primer quintil en el total de ingresos nacionales en 2007 era $1.93 \%$, la más baja del continente. El valor del coeficiente de $\mathrm{GINI}^{5}$ para Honduras fue superior al 0.50. El informe de la CEPAL destaca que "la disminución de la pobreza en la región fue impulsada en parte por las mejoras distributivas en un período 20022008", tiempo en el cual el índice de Gini, en los países latinoamericanos y del caribe, se redujo en promedio en $5 \%$. Honduras es el país con el más alto índice de desigualdad entre los cuatro que no han conocido una mejora redistributiva y ello puede ser una de las razones por las cuales la reducción de la pobreza se ha dado de una forma tan paulatina. (Ver tabla No. 2)

\subsection{Resultados del estudio determinantes de la culminación a tiempo en educación.}

La culminación de la educación primaria es un objetivo clave y básico de los sistemas educativos en países de menor desarrollo es por ello, el interés

\footnotetext{
5 El coeficiente de Gini mide la desigualdad en los ingresos. Es un numero entre 0 y 1 , donde Cero $(0)$ corresponde a la igualdad perfecta (todos tienen los mismos ingresos) y 1 corresponde a la desigualdad perfecta (una persona tiene todos los ingresos y los demás ninguno)
}

de emprender mediciones de la dinámica de los indicadores involucrados, partiendo de los hallazgos encontrados en estudios universales, los cuales han explorado de forma rigurosa los determinantes de la educación y su avance. A continuación, los principales resultados encontrados, de forma que estos constituyan una fuente de referencia, para el mejor entendimiento y desarrollo del tema.

En el caso del ODM 2A, se desestima el uso del indicador oficial (tasa neta de matriculación en la enseñanza primaria) por considerar que la tasa de culminación a tiempo en la educación es un indicador más robusto del grado de impacto de las políticas públicas en formar capacidades en la población, comparado con la simple matricula. Para calcular la tasa de culminación a tiempo, se buscan los factores que determinan:

- La tasa de entrada al primer grado de la primaria de niños y niñas a tiempo (en la edad cuando deben entrar en el primer grado);

- Las tasas de promoción de grado en cada ciclo educativo; y

- Las tasas de continuación desde la primaria y de la secundaria al ciclo siguiente. 
Según Rosales (2006), la educación es un proceso de decisiones secuenciales. Sí se parte de que todos los individuos asisten a la escuela primaria, los próximos niveles de decisión serían: Completar la primaria, continuar de la primaria a la secundaria básica, completar la secundaria, continuar al nivel medio superior, completar la media superior y asistir y completar la educación terciaria.

El razonamiento de Rosales parecería lo ideal, pero según estadísticas actuales de muchos países y en el caso específico de Honduras, existen muchas discrepancias que restringen el logro efectivo de la continuación y culminación de la educación desde la educación primaria hasta el último nivel superior de estudios. Según el informe de EFA (2013) revela que los indicadores de repitencia de primero, segundo y tercer grado son preocupantes dado que representaron en su orden el 9.53\%, 6.32\% y $5.1 \%$ respectivamente para 2012 , y la deserción a 2013 refleja el $1.06 \%$ y hacia 2015 se estimó que oscilo en 1\%. Respecto a la matrícula existió una brecha aún del $31.13 \%$ y para finalizar el acceso a la preparatoria una brecha hacia 2015 de $9.34 \%$. Con estos resultados, no se desestimó que la continuidad y la culminación de la educación primaria seguirán siendo metas para el país, ya que, al efectuarse por completo dichos retos, garantizarán la continuidad de estudios superiores y con esto superar la pobreza y avanzar hacia una mayor equidad de oportunidades.

En respuesta al cumplimiento del objetivo universal, el aumento de los niveles educacionales, en especial los de los grupos más pobres, así como la disminución en brechas de género, permitirán incrementar la movilidad social y elevar los retornos a la educación y a la calidad misma de los recursos humanos. Es por ello, que es importante analizar el logro de la culminación a tiempo de los estudios primarios, para garantizar la continuidad a niveles superiores de educación.

Estudios como los de Sabonge (2004) muestran que la asistencia escolar de los niños se asocia positivamente con el nivel educativo de los padres. Es así como los padres con mayor educación tienden a valorar más la educación para sus hijos, como producto de ello tienen mayores ingresos que les permite mantener a sus hijos en el sistema educativo reduciendo así la probabilidad de que ellos trabajen. Esta premisa confirma que la presencia de ambos padres en el hogar tiene una relación directa en el bienestar de sus hijos, permitiéndoles continuidad en el sistema educativo.

Por otro lado, encuentra que el tamaño del hogar hondureño tiene un efecto negativo sobre la probabilidad de asistir a la escuela por diversos factores, primando entre ellos la falta de recursos, en respuesta a esta falencia los hogares de zonas rurales ven a los niños como mano de obra disponible para incorporarlos a actividades del sector primario y respecto a la zona urbana estos son vistos para insertarlos en el mercado laboral informal, y como producto de ello conlleva a sumar más niños con altos porcentajes de discontinuidad escolar y considerables tasas de repitencia.

Concatenado al trabajo de Sabonge, Morales (2004) analizaron sí la educación de los padres incide en la elección de los jóvenes de estudiar/ trabajar, en particular cuestionan sí es posible que padres con bajos niveles educativos pueden valorizar la educación de sus hijos, y responden que esta valorización llevaría a las autoridades a elaborar programas que sin buscar elevar el nivel educativo de los adultos, busquen incrementar su interés por la educación de sus hijos mostrándoles las ventajas que ello tiene. Además, revelaron la importancia que tiene el ingreso monetario de los padres en la elección de los hijos y confirma que es un resultado recurrente en muchos países, y que la forma de enfrentar el problema es otorgar subsidios focalizados a la educación, para tener un rendimiento efectivo de los recursos.

El trabajo de Vos y Ponce (2004) apuntan a tres factores con el mayor impacto sobre la matriculación de niños en las escuelas de Ecuador: (a) mejorar la calidad de los docentes, reduciendo así su número; (b) usar los ahorros para invertir en la infraestructura educativa, subsidies, e incentivos a los más pobres; $y$ (c) direccionar bonos y otros incentivos monetarios que tengan un efecto sobre la matriculación y atención escolar. El estudio también apunta a los beneficios de descentralizar las decisiones educacionales y dar más poder a las comunidades locales para elegir docentes y directores. 
Lo anterior induce a definir una métrica que permita establecer el grado de verosimilitud del logro de la culminación de la educación primaria, considerando las políticas educativas vigentes y las trayectorias educativas actuales de la población en edad de asistir a la primaria. Así también modelos que reporten la probabilidad de terminar exitosamente los estudios en la etapa de escolarización de los niños en edad de asistir a la educación primaria/secundaria. Teniendo en cuenta no los grados que están siendo cursados, sino los efectivamente aprobados, en relación con la duración teórica del nivel de educación primaria/secundaria.

El monitoreo de la meta de la finalización de un ciclo completo de enseñanza primaria se utilizan los indicadores de tasa neta de matriculación en la enseñanza primaria; proporción de alumnos (as) que comienzan el primer grado y llegan al sexto grado; y la tasa de alfabetización de personas entre 15 y 24 años. Para verificar la igualdad entre los sexos en los niveles educativos primario y secundario se utiliza la relación entre niños y niñas en la enseñanza primaria, secundaria y superior; la tasa de cobertura escolar por dominio y sexo; proporción de mujeres entre los empleados remunerados en el sector no agricola; ingresos mensuales por sexo, dominio y nivel educativo y la proporcion de escaños ocupados por mujeres por en el Congreso Nacional.

\subsubsection{Estimaciones econométricas de los determinantes de la culminación en tiempo}

Con estos insumos se construyeron los determinantes de la matrícula en el primer año de la primaria, de la aprobación de grado en la primaria, y de completar el ciclo y continuar al siguiente. Los determinantes de estos tres objetivos se describen a continuación:

\section{Gasto público por estudiante:}

El gasto público en educación es la variable de política directamente relacionada con los resultados en educación. Para este determinante se utilizó información de gasto del gobierno por nivel educativo (Primaria/Secundaria) para los años 2010 y 2011, la cual fue facilitada por la Secretaría de Finanzas de Honduras, convalidado por la Secretaria de Educación. Se espera una relación positiva con las tasas de matriculación, promoción, graduación y continuación.

\section{Nivel de Infraestructura Pública:}

El nivel de infraestructura pública captura los demás componentes del gasto público que puedan tener un impacto sobre la educación (carreteras, electricidad, telefonía, etc.). Para este determinante se utilizó un proxy, en vista de que no se contaba a nivel de departamento con este ítem de gasto, es así como se utilizó el nivel de acceso a energía eléctrica como variable cercana, capturada por la EPHPM consolidada con las rondas de mayo-septiembre.

\section{Consumo per cápita de los Hogares:}

El consumo o el ingreso, medidos a nivel del hogar, captura la capacidad del hogar de acceder a servicios de educación. Se tomó como proxy la variable ingreso per cápita de los hogares, capturada mediante la EPHPM consolidada.

\section{Tasa de Mortalidad (menores de 5 años):}

La mortalidad infantil tiene una relación obvia con la educación, impactando de forma negativa a todas las variables dependientes. Esta tasa es provista por estimaciones y cálculos del ODM4, que refiere a Mortalidad Infantil.

\section{Otros Determinantes:}

Otros controles en los modelos estimados incluyen: el dominio de residencia (urbano / rural); el sexo del niño; si el piso o el techo es "mejorado"; el nivel educativo del jefe del hogar; el quintil del ingreso del hogar; si el niño atendió la preescolar el año anterior; y la orfandad de padre o madre.

Revista Economía y Administración (E\&A) / VOL. 8 


\section{Premia de salario:}

Por último, también se considera el efecto del premio salarial por haber completado un ciclo educativo. Para estimar este efecto se necesita datos de la premia salarial por completar cada ciclo educativo, proxy del incentivo de adquirir la educación. Una forma simple de aproximar este dato es computar el salario promedio de trabajadores con el ciclo completo, en relación con el salario promedio de los trabajadores sin haber completado el ciclo. Un problema que ocurre con esta metodología es que hay presencia de sesgo de selección en vista que se compara el salario promedio de personas ocupadas vs características de personas que pueden o no cumplir con esta condición. El sesgo se estima mediante los determinantes de los salarios solamente para la población que está trabajando dado que estos tienen un salario más alto comparado al salario esperado de los que no están trabajando. Es justamente por esta diferencia (y el salario de reserva implícito) que se genera un sesgo, en vista de ello se procedió a corregir las estimaciones por sesgo de selección existente utilizando el procedimiento de Heckman, de forma que los coeficientes resultantes sean consistentes y robustos.

\subsubsection{Estimación de la premia de salario}

En lo que refiere al premia al salario se utilizó como fuente básica, la especificación de modelos de retornos marginales a la educación de López-
Méndez María (2013). Este determinante incluye las estimaciones de un modelo econométrico que explica la decisión de invertir en educación (o capacitación) desde un punto de vista puramente económico.

Se asume que la persona decidirá estudiar (o capacitarse) si los beneficios superan a los costos. En este sentido se analiza el salario adicional que un ocupado obtendría con la finalización de los diferentes niveles de enseñanza formal, y con la capacitación que le permitiría ejercer puestos laborales con mayores niveles de complejidad. Este punto resulta sumamente relevante para analizar las "señales" que otorga el mercado laboral en los de la decisión de invertir en capital humano.

La estimación de retornos marginales a la educación para el caso de Honduras se analizó en función a la teoría de capital humano por el lado de la oferta bien considerando los estudios de Mincer, y por el lado de la demanda se consideró el planteamiento de Trurow, quien expone que tanto los salarios y la productividad de un individuo están más asociados al puesto de trabajo que a las personas, en vista de que no se opera en mercados competitivos. Dado los dos enfoques anteriores, se consideró una ecuación de ingresos que relaciona el logaritmo del salario laboral respecto a un conjunto de características individuales que podrían afectar la productividad marginal del individuo. Entre dichas variables se encuentran las relacionadas con el nivel educativo alcanzado; experiencia, edad, sexo, sector laboral, entre otras variables.

El siguiente modelo fue estimado para cada departamento regional en Honduras:

$$
\begin{aligned}
\operatorname{Ln}(w)_{i k t}=\beta_{0} & +\sum_{i=1}^{3} \beta_{i}(\text { Nivel Educativo })+\beta_{4} \text { experiencia }+\sum_{i=5}^{7} \beta_{i}\left(\sum_{j=18}^{44} \text { ed }\right)+\beta_{8} \text { sexo } \\
& +\beta_{9} \text { emplpubli }+\beta_{10} \text { emplpriv }+\beta_{11} \text { cuentaprop }+\beta_{12} \lambda_{i}+e_{i}
\end{aligned}
$$


Donde $\operatorname{Ln}(w)_{i k t}$ es el logaritmo del salario del individuo $i$ en el departamento $k$ para el año $t$. Para el nivel educativo alcanzado, se consideraron 3 niveles (Primaria Completa, Secundaria Completa, Superior Completa). La experiencia constituye el nivel de experiencia en el mercado laboral. La variable reporta la cohorte estudiado en el modelo de 18-44 años. Las variables sexo, emplpubli, emplpriv, y cuentaprop representan respectivamente: el sexo del agente estudiado, los agentes que trabajan en el Sector Público, los trabajadores del Sector Privado, y los individuos que laboran de forma independiente.

La variable $\lambda$ es el ajuste por sesgo de selección.
El término de error, se compone por variables no observables que inciden sobre la remuneración como retorno a la educación. Los coeficientes estimados $\beta_{1}, \beta_{2}, \beta_{3}$ son los premios salariales que buscamos por tener primaria, secundaria, $\mathrm{y}$ superior completa, respectivamente.

Se consideraron únicamente a los trabajadores asalariados, ya que las bases de datos no permiten separar los retornos provenientes del trabajo y del capital, de principio se contó con una muestra de 14,119 hogares, de los cuales se obtuvo información de 66,156 personas y del total de éstas, el 34\%\% son mujeres jefas de hogar y el $66 \%$ son hombres jefes de hogar.

Modelo de Retornos a la Educación

\begin{tabular}{|c|c|c|c|c|c|c|c|c|c|c|c|c|c|}
\hline $\ln (w)$ & pric & secc & supc & ed1824 & ed2534 & ed3544 & exper & sex & emppubl & emppriv & depdom & lambdai & cons \\
\hline Coefficient & $0.4285^{* * *}$ & $0.6463^{* * *}$ & $0.6911^{* * *}$ & $0.2828^{* * *}$ & $0.4156^{* * *}$ & $0.3972^{* * *}$ & $0.0238^{* * *}$ & $-0.1161^{* * *}$ & $1.9252^{* * *}$ & $1.6703^{* * *}$ & $-0.0120^{* * *}$ & $3.4124^{* * *}$ & $5.3689^{* * *}$ \\
\hline Std.Err. & 0.0214 & 0.0187 & 0.0297 & 0.0249 & 0.0218 & 0.0229 & 0.0008 & 0.0236 & 0.1015 & 0.1072 & 0.0008 & 0.2785 & 0.1050 \\
\hline $\mathrm{N}$ & 10872 & & & & & & & & & & & & \\
\hline$\|$ & -12000 & & & & & & & & & & & & \\
\hline
\end{tabular}

Figura No.3: Modelo de Retornos a la Educación

Fuente: Datos tomados de la EPHPM, con muestra consolidad de las rondas de mayo y septiembre de 2011, INE, Honduras.

Obsérvese que todos los coeficientes son estadísticamente significativos, excepto para la variable cuenta propia que finalmente no fue incluida. Se ha realizado también los test de nulidad de estos coeficientes en ambas ecuaciones (sesgada e insesgada) simultáneamente proporcionando un resultado similar. Puede verse que los signos de los coeficientes son los esperados y así también las magnitudes de las variables consideradas

Utilizando los coeficientes corregidos por sesgo de selección, podemos estimar el retorno marginal a la educación según ciclo educativo. Estos son calculados respecto del menor nivel educativo (primario completo). Por lo tanto, cada retorno debe leerse como "el cambio porcentual en el ingreso por hora trabajada que debería esperarse para un individuo que aumenta su educación desde primaria completa a la categoría educativa analizada".

\subsection{Resultados de los modelos}

Utilizando los premios salariales estimados, junto con la información de los hogares y del gasto público en educación e infraestructura, se pueden estimar los modelos logísticos para analizar los determinantes de la matriculación en la primaria, de la promoción de grado en cada ciclo, y de la graduación y continuación al siguiente ciclo educativo.

Tomando en cuenta estudios previos como los de Sánchez \& Sbrana (2009; 2010), (Ponce, Bedi, and Vos 2003), y (Vos and Ponce 2004) se consideraran las siguientes variables como determinantes para el análisis con un modelo logístico:

- Gasto per cápita en educación de 2010

- Ingreso per cápita del hogar 
- Acceso a electricidad en los hogares

- Mortalidad infantil

- Retornos a la educación

- Las variables de control (sexo, características del hogar, quintil de ingreso, etc.)

La variable Acceso a Electricidad en los Hogares (electhog) juega el rol de proxy de la variable "Infraestructura Pública", por otro lado, la variable mortalidad infantil y en la niñez se diseñó por regiones sanitarias, en vista que solo con esos estratos se cuenta para dicha información. También se contó con datos de gasto público en educación por nivel educativo y por departamento, en el caso de los departamentos no se toma en cuenta los departamentos de Gracias a Dios e Islas de la Bahía en vista de que la EPHPM no tiene cobertura en dichos departamentos por la distancia y los altos costos de movilización.

En vista de que muchos estudios indican que la educación de los padres incide fuertemente en la probabilidad de matrícula, asistencia y culminación de los estudios de los niños, se consideró la variable educación del jefe del Hogar. También se consideró la variable quintil del ingreso del hogar de forma que el diseño reporta consistencia en la distribución del ingreso de los individuos de manera más uniforme que un promedio. No obstante, se crearon variables complementarias como "huérfanos de padre", "huérfanos de madre", "huérfanos de ambos", dado que la presencia de los padres en el hogar influye en la asistencia y culminación de los niños en el ciclo básico. Se consideró la pregunta que "si el niño atendió preescolar el año anterior", indicador coadyuvante de la probabilidad de matriculación en primaria. Respecto a otras condiciones del hogar se consideró la variable dummy de "piso y techo mejorado" como indicador de condiciones de acceso a infraestructura básica en el hogar.

Los resultados informan que los modelos logísticos estimados, que son la base de análisis para el efecto marginal estimado más abajo ${ }^{6}$.

\footnotetext{
6 Los coeficientes reportados en los modelos logísticos no tienen una interpretación clara. Esto es todavía más complicado por incluir interacciones entre distintas variables. Por ejemplo, para controlar por la posibilidad de que el ingreso tenga un efecto distinto en el quintil
}

Como esperado, se observa una dificultad en estimar una relación estadística robusta entre los determinantes y las variables dependientes. En varios casos los signos no son los esperados, y las correlaciones no son significativas. Pero puede verse claramente que, cuando el coeficiente es significativo, el signo tiende a ser el esperado. Los modelos también son sensibles a cambios en la especificación, algo que se espera debido a la baja variabilidad de algunos indicadores y otras dificultades en construir la base de datos para el análisis.

Los test de especificación de los modelos reflejan estos problemas. En el caso de la estimación de los determinantes del ingreso a la primaria y de la graduación y continuación de la primaria a la secundaria, los modelos están bien especificados? Los modelos de promoción en la primaria y en la secundaria demuestran algunos problemas de especificación. En el caso de la tasa de promoción en la primaria, el modelo tiene una baja capacidad de explicar la variabilidad en las tasas de promoción. En el caso de las tasas de promoción en la secundaria, el modelo tiene algunos problemas de especificación, pero se aproxima bien a los datos observados.

\section{Elasticidades}

Respecto a las elasticidades calculadas, tenemos que él gasto per cápita en educación tiene efectos distintos en cada ciclo. Interesante que, en la secundaria, un aumento en el gasto para este ciclo per cápita se ve asociado con una reducción en la tasa de promoción, algo que se puede explicar si la causalidad es la opuesta, tal como si el gasto en educación responda a problemas de logros educativos. Otros estudios también han estimado elasticidades con el signo opuesto al esperado y no significativas, algo que se podría explicar

más bajo, se opta por estimar esta interacción. El impacto del ingreso no se puede medir por la magnitud, ni la significancia, del coeficiente reportado. Se requiere estimar el efecto marginal de estas variables, lo cual lleva en cuenta la interacción.

7 Para examinar la especificación de las variables, se busca tener un modelo en lo cual no hay ninguna variable independiente excluida que sea significativa (linktest en Stata). Para medir la precisión del modelo en relación a los datos ("goodnes-of-fit"), medimos la capacidad del modelo de identificar valores positivos correctos en proporción al total de valores positivos en realidad, y la proporción de valores positivos falsos en proporción a los valores negativos en realidad (Iroc en Stata). Otro teste para medir la precisión del modelo es el Hosmer-Lemeshow, que informa si la frecuencia prevista por el modelo se aproxima a la frecuencia observada en los datos. 
por diferencias importantes entre las decisiones de la población rural y urbana, además de la demanda por escuelas públicas y privadas entre los pobres y no pobres. Ponce, Bedi, y Vos (2003) y Vos y Ponce (2004), por ejemplo, encuentran que la importancia de cada determinante de la tasa de matriculación en Ecuador varía entre el ciclo básico y la secundaria. Los autores también identificaron diferencias importantes en el proceso de decisiones de los hogares urbanos comparado a los hogares rurales, y de los hogares pobres comparados con los no-pobres.

La tasa de mortalidad infantil no es significativa en el ingreso o en la tasa de promoción. Si tiene el efecto esperado en la graduación y continuación de la primaria hasta la secundaria. El premio salarial estimado tiene el efecto esperado. Un aumento de $1 \%$ en el premio salarial resulta en un aumento de entre $1 \%$ y $2 \%$ en la probabilidad de graduación, ingreso y promoción. Un aumento de $1 \%$ del ingreso per cápita del hogar aumenta la probabilidad de matriculación en un $25 \%$ y es el principal determinante en la probabilidad de matriculación, en vista que es una de las principales restricciones de los hogares de ingresar los niños a la educación primaria

\section{CONCLUSIONES Y RECOMENDACIONES}

Los resultados demuestran que las mejoras en las condiciones socioeconómicas de los hogares en Honduras tienen un efecto importante sobre los logros educativos en el país. Políticas públicas que resulten en aumentos del ingreso, aumentos en el premio salarial, y mejoras en la infraestructura pública mejoran las tasas de matrícula, promoción, y graduación de los estudiantes. Esta combinación resulta en mejoras en la tasa de culminación a tiempo en la educación, un indicador más robusto de las capacidades en la población en comparación comparado con la simple matricula.

El gasto no incide en la decisión de matricular, pero sí afecta las tasas de promoción. La disponibilidad de infraestructura tiene su mayor impacto sobre la graduación y continuación al siguiente ciclo educativo. El ingreso per cápita del hogar, indicador del poder adquisitivo disponible para la educación, tiene el mayor impacto sobre la matricula del alumno a tiempo. El premio salarial por tener una educación completa incide en todos los resultados educativos. Este último, por su efecto en el ingreso a la escuela, la promoción de año, y la graduación y continuación, debe indicar una oportunidad para estimular las políticas del mercado laboral para influenciar un importante objetivo de desarrollo para Honduras.

\section{AGRADECIMIENTOS Y RECONOCIMIENTOS}

La presente investigación es la versión original de la investigación, la versión en inglés es traducción de algunos fragmentos de ésta, la cual fue publicada en la página del Department of Economic and Social Affairs (DESA) de Naciones Unidas.

http://www.un.org/en/development/desa/policy/capacity/presentations/honduras/Determinantsof-EDUC-in-Honduras-english.pdf 
ANEXOS TABLA No.3: Determinantes de la culminación a tiempo en la educación (Regresión)

\begin{tabular}{|c|c|c|c|c|}
\hline Variables & $\begin{array}{c}\text { Ingreso a la } \\
\text { Primaria }\end{array}$ & $\begin{array}{l}\text { Promoción en } \\
\text { Primaria }\end{array}$ & $\begin{array}{l}\text { Promoción en } \\
\text { Secundaria }\end{array}$ & $\begin{array}{c}\text { Graduación de } \\
\text { primaria }\end{array}$ \\
\hline Gasto público p.c.- primaria (logaritmo) & -0.580 & $1.148^{* *}$ & & 0.259 \\
\hline Gasto público p.c.- secundaria (logaritmo) & & -0.331 & $-0.517 * *$ & \\
\hline Tasa de Mortalidad Infantil & 0.001 & $-0.145 * *$ & -0.002 & $-0.024 *$ \\
\hline Tasa de Mortalidad Infantil al cuadrado & & $0.003 * *$ & & \\
\hline Premio salarial - primaria & $0.666 * * *$ & 0.092 & & $0.624 * *$ \\
\hline Premio salarial - primaria al cuadrado & & $0.580 * *$ & & \\
\hline Premio salarial - secundaria & & & 0.373 & \\
\hline Premio salarial - secundaria al cuadrado & & & -0.130 & \\
\hline Ingreso del hogar p.c. (logaritmo) & 0.195 & $0.148^{* *}$ & 0.009 & \\
\hline Ingreso del hogar p.c. & & & & 0.001 \\
\hline \multicolumn{5}{|l|}{ Quintil de ingreso $($ base $=$ quintil 1$)$} \\
\hline Quintil 2 & $-8.735^{*}$ & & & 0.200 \\
\hline Quintil 3 & -7.649 & & & -0.647 \\
\hline Quintil 4 & $-15.516^{* *}$ & & & 1.540 \\
\hline Quintil 5 & 1.298 & & & $1.726^{* *}$ \\
\hline \multicolumn{5}{|l|}{ Ingreso per cápita (ln) por quintil } \\
\hline Ingreso en quintil 2 & $1.281 *$ & & & \\
\hline Ingreso en quintil 3 & 0.931 & & & \\
\hline Ingreso en quintil 4 & $1.896^{* *}$ & & & \\
\hline Ingreso en quintil 5 & -0.261 & & & \\
\hline \multicolumn{5}{|l|}{ Ingreso per cápita por quintil } \\
\hline Ingreso en quintil 2 & & & & -0.000 \\
\hline Ingreso en quintil 3 & & & & 0.000 \\
\hline Ingreso en quintil 4 & & & & -0.001 \\
\hline Ingreso en quintil 5 & & & & -0.001 \\
\hline Hogar tiene servicio eléctrico & $-0.433 * *$ & $0.443 * *$ & -0.026 & $1.025^{* *}$ \\
\hline Niño atendió preescolar & $2.068 * * *$ & & & \\
\hline Niño es hombre & -0.175 & $-0.370 * *$ & $-0.469 * *$ & -0.202 \\
\hline Niño es huérfano de madre & -1.001 & -0.086 & -0.560 & \\
\hline Niño es huérfano de padre & 0.438 & $-0.270^{*}$ & 0.062 & \\
\hline Personas por habitación & & $-0.161 * *$ & $-0.151 * *$ & \\
\hline Constante & 4.374 & $-3.770 *$ & $7.227 * *$ & -1.873 \\
\hline$N$ & 1,696 & 10,149 & 3,866 & 1,413 \\
\hline
\end{tabular}


TABLA No.4: Elasticidades de los determinantes de la culminación a tiempo en la educación (Regresión)

\begin{tabular}{lcccc}
\hline \multicolumn{1}{c}{ Variables } & $\begin{array}{c}\text { Ingreso a la } \\
\text { Primaria }\end{array}$ & $\begin{array}{c}\text { Promoción en } \\
\text { Primaria }\end{array}$ & $\begin{array}{c}\text { Promoción en } \\
\text { Secundaria }\end{array}$ & $\begin{array}{c}\text { Graduación de } \\
\text { primaria }\end{array}$ \\
\hline Gasto público p.c.- primaria o secundaria & \multicolumn{1}{c}{-0.145} & $0.117^{* *}$ & \multicolumn{1}{c}{$-0.055^{* *}$} & 0.057 \\
\hline Mortalidad Infantil & 0.008 & 0.025 & -0.006 & $-0.157^{*}$ \\
Premio salarial por completar el ciclo & $0.020^{* *}$ & $0.020^{*}$ & 0.012 & $0.010^{* *}$ \\
Ingreso del hogar p.c. & $0.248^{* *}$ & $0.015^{* *}$ & 0.001 & 0.092 \\
\hline Hogar tiene servicio eléctrico & $-0.102^{* *}$ & $0.049^{* *}$ & -0.003 & $0.278^{* *}$ \\
\hline $\mathrm{N}$ & 1,696 & 10,149 & 3,866 & 1,413 \\
LL & -802.168 & $-3,221.231$ & $-1,291.165$ & -659.436 \\
LR chi2 & 300.904 & 257.004 & 45.261 & 173.051 \\
Prob $>$ chi2 & 0.000 & 0.000 & 0.000 & 0.000 \\
Pseudo R2 & 0.158 & 0.038 & 0.017 & 0.116 \\
\hline
\end{tabular}

$+p<0.1 ; * p<0.05 ; * * p<0.01$

\section{ANÁLISIS:}

Los resultados tienen importantes limitaciones estadísticas. La estimación de las elasticidades en este estudio tiene por su principal objetivo la retroalimentación de los coeficientes necesarios para el modelo MAMS. Debido a que el MAMS ya tiene definido su sistema de ecuaciones interna, esta limitación requiere una formulación rígida de los modelos. Se necesita que las elasticidades del gasto en la educación, la infraestructura pública, la mortalidad infantil, el premio salarial, y el ingreso de los hogares. Para mejorar los resultados estadísticos se requiere datos con un mayor nivel de detalle. Por ejemplo, mejores estimadores del gasto público en infraestructura que capturen directamente el stock y el flujo de inversiones que sirvan para mejorar el acceso a los centros de educación.

Asímimo, los resultados demuestran que, si bien el rendimiento de la educación y el valor que otorga el mercado laboral a las capacidades adquiridas, debería ser un aspecto importante para planificar la política pública, existen beneficios sociales o externos adicionales relacionados con la educación que deberían considerase para realizar una eficiente asignación de recursos. Tales beneficios pueden relacionarse con mayores tasas de crecimiento de las unidades económicas regionales, menores tasas de desempleo y pobreza que experimentan las personas mejores capacitadas, con los beneficios intergeneracionales existentes en la educación (los hijos de padres con mayores niveles de estudios crecen con mejores necesidades cubiertas) entre otros aspectos destacables.

La importancia de este estudio va más allá de las estimaciones de las elasticidades. Cualquier trabajo de rigor que busca informar la política pública para lograr los objetivos económicos, sociales, y de desarrollo humano de un país requiere de un buen entendimiento del impacto que estas podrían tener. Estas elasticidades son difíciles de estimar, en parte debido a la dificultad de modelar la complejidad de la interacción, aunado a las falencias de no contar con datos detallados para algunas variables, como se mencionó en apartados anteriores. La primera parte se puede avanzar a medida que se diseñan y se desarrollan los modelos econométricos, pero es importante mencionar que la falta de datos requiere de inversión real y dedicación institucional por parte del gobierno para crear las bases de datos necesarias. Este problema no es simple de resolver. Una recomendación para optimizar el uso de las herramientas de estimación y previsión sería recopilar más información de precios, gasto, consumo, y más detalles demográficos y socio económicos a nivel de los individuos. 
ISSN 2219-6722 / ISSNE 2222-2707

Economía y Administración (E\&A) , Vol.8 (2) pp.79-97 @ 2017, IIES-UNAH MARÍA AUXILIADORA LÓPEZ MÉNDEZ / MARCELO T. LAFLEUR
MÁn de

En gran parte con el desarrollo de las encuestas de hogares se ha aumentado la disponibilidad de datos. Pero al mismo tiempo, se requiere también de información detallada sobre la política pública, más específicamente niveles de gasto e inversión por departamento y por municipio en acceso a salud, educación y saneamiento. Para ampliar más este punto se encuentra que en Honduras, la dificultad para obtener datos sobre las políticas direccionadas a la mortalidad infantil más detalladas que el gasto a nivel del programa de salud, limita la utilidad de este análisis. No es suficiente tener datos de gasto público como generalidad, sólo si estos permiten agrupar gastos y ejecuciones de políticas para reducir la mortalidad infantil. Otros insumos de mucha utilidad para este análisis serían datos de gasto público y del stock de infraestructura (carreteras y tubería instalada), del acceso efectivo a centros de atención (la distancia de cada hogar), y otros que permitiesen un análisis más preciso del efecto de las políticas y los resultados en la salud. Estudios de caso que logran detallar interacciones entre políticas y resultados difícilmente pueden ser generalizados o replicados para informar estrategias nacionales.

Enun mundodonde el impacto delas intervenciones públicas tiene un efecto reducido al aproximarse del objetivo de política, y bajo un espacio fiscal que se ve reducido últimamente, se torna cada vez más importante aumentar la eficiencia del gasto público. La identificación de los canales más efectivos para lograr los objetivos de desarrollo humano requiere datos cada vez más detallados. El análisis hecho para Honduras demuestra que usar el gasto público como instrumento de política en su totalidad o en cortes más específicos no es una estrategia eficiente para lograr resultados de desarrollo humano. Es por ello por lo que es mejor diseñar intervenciones más específicas e informadas, que arrojarán resultados mucho más eficientes y reducirán el impacto presupuestario. 
Determinantes de las Metas de

\section{REFERENCIAS}

BCH (2013). Memorial Anual 2012. Tegucigalpa

BCH. (2012). Honduras: Tasa de politica Monetaria. Tegucigalpa.

BCH. (2012). Indice de Precios al Consumidos Julio 2012. Tegucigalpa.

BCH. (2012). Indice Mensual de Actividad Económica Junio 2012. Tegucigalpa.

BCH. (2012). Informes varios. Tegucigalpa.

BCH. (2012). Programa Monetario 2012-2013. Tegucigalpa.

BCH. (2012). Resumen Ejecutivo Semanal 16 de agosto de 2012. Tegucigalpa.

BCH. (Junio 2012). Comercio Exterior de Mercancias Generales. Tegucigalpa: $\mathrm{BCH}$.

Castellanos, P. (2005). Evolución Históric a de la Salud en Honduras durante el Siglo XX. Revista Medica de Honduras 73 (Suplemento 2) , 17-36.

CEPAL. (2012). Balance Preliminar de las Economías de América Latina y del Caribe 2012. NY: Naciones Unidas.

CEPAL. (2012). Informe Macroeconómico Junio 2012 Honduras. Versión no editada. México: Comisión Económica para América Latina y el Caribe.

CEPAL. (2011). Panorama Social de América Latina 2010. Naciones Unidas.

Charmarbagwala, R.; Ranger, M.; Waddington, H. y White, H. (2004), The determinants of child health and nutrition: a meta-analysis. International Evaluation Group Working Paper. Eashington, D.C.: The World Bank

GoH y SNU (2010), Objetivos de Desarrollo del Milenio Honduras 2010. Tercer Informe de País, San José, Costa Rica.

INE (mayo 2011), Encuesta Permanente de Hogares de Propósitos Múltiples, mayo 2011,
Tegucigalpa, INE.

INE (mayo 2012), Encuesta Permanente de Hogares de Propósitos Múltiples, mayo 2011, Tegucigalpa, INE.

INE. (Mayo 2013), Encuesta Permanente de Hogares de Propósitos Múltiples, publicación, recuperado de, http://www.ine.gob.hn/ Documentos/Hogares\%202013/resumen\%20 ejecutivo $\% 20$ mayo $\% 202013$.pdf.

Kaldewei, C. y Pitterle, I. (2011), Behavioral factors as emerging main determinants of child mortality in middle-income countries: a case study of Jordan. New York: DESA Working Paper no. 103.

Larson, B.; Minten, Bart y Razafindralambo, Ramy (2006). Los vínculos entre los Objetivos de Desarrollo del Milenio para reducir la pobreza, educación, acceso al agua y los hogares el uso del agua en los países en desarrollo: evidencia de Madagascar.

LaFleur, Marcelo T, (2014) "Determinantes del acceso a fuentes de agua y saneamiento mejorados y los Objetivos de Desarrollo del Milenio en Honduras, United Nations, New York, USA.

LaFleur, Marcelo T, (2014) "Determinantes del acceso de la salud materna e infantil y los Objetivos de Desarrollo del Milenio en Honduras, United Nations, New York, USA

López- Méndez, María; (2013) "Evolución Temporal de los Retornos Marginales a la Educación de Honduras", 1er CEAT, IIESUNAH, Honduras.

Morales, Rolando. (2004), Asistencia escolar y participación laboral en Honduras. Un modelo biprobit. White Paper, PNUD, Honduras.

O’Donnell, O.; van Doorslaer, E.; Wagstaff, A.; Lindelow, M. (2008), Analysisng health equity using households survey data: a guide to techniques and their implementation. Washingnton, D.C.: The World Bank 
Pacheco, J. (2012), Determinantes socioeconómicos de la educación, la mortalidad $y$ el acceso al agua potable y el saneamiento: un análisis econométrico, PNUD y UN-DESA

Ponce (2012), Determinates del acceso y la permanencia en la educación, la mortalidad de la niñez y materna y el acceso a servicios básicos de agua y saneamiento: Evidencia para el Estado Plurinacional de Bolivia, Quito: FLACSO-Ecuador

Ponce, Juan, Arjun Bedi, and Rob Vos. 2003. “CCómo hacer más eficiente el gasto educativo?” ¿In Quién se beneficia del gasto social en el Ecuador?: desafios para mejorar la calidad y eficiencia del gasto social, by Rob Vos, 89-118. Unidad de Información y Análisis, SIISE de la Secretaría Técnica del Frente Social.

Rosales, Susset. 2006. "Influencia De Variables Socioeconómicas En El Proceso Educativo."

Sabonge, Kenia. (2004) "Factores Asociados a la asistencia escolar en la población de 5 a 19 años. Honduras 2003, Universidad de Costa Rica.

Sánchez, Marco V., and Giacomo Sbrana. 2010. "Determinants of Education Attainment and Development Goals in Yemen". Department of Economic and Social Affairs.

Sánchez, Marco V., and Giacomo Sbrana. 2009. "Determinants of Attendance in Jordan's Education."

Sánchez, Marco V., and Rob Vos. 2010. "Latin America and the Caribbean's Challenge to Reach the MDGs: Financing Options and Trade-offs." DESA Working Paper 74 (May).

SDP, INE, SESAL e ICF (2013), Encuesta Nacional de Demografía y Salud ENDESA 20112012.

Secretaría de Salud. (2002). Análisis de Mortalidad Materna año 2002 Honduras. . Tegucigalpa.

SEFIN. (2012). Cifras Fiscales Mayo de 2012. Tegucigalpa.
Sistema de la Naciones Unidas en Honduras (2010). Objetivos de Desarrollo del Milenio, Honduras 2100

Torres, S., \& Sequeira, S. (2003). Investiogación Cualitativa sobre la Mortalidad Materna en Honduras. Honduras: Instituto Nacional de la Mujer (INAM).

UNICEF. (Diciembre de 2012). Honduras . Recuperado el 30 de Enero de 2013, de Unicef: http://www.unicef.org/honduras/14352_24064. htm

Vos, Rob, Marco V. Sánchez, Enrique Ganuza, Hans Lofgren, and Carolina Díaz-Bonilla, ed. 2010. Public Policies for Human Development: Achieving the Millennium Development Goals in Latin America. New York: Palgrave Macmillan.

Vos, Rob, Marco V. Sánchez, and Cornelia Kaldewei. 2008. "Latin America and the Caribbean's Challenge to Reach the MDGs: Financing Options and Trade-offs." DESA Working Paper 68 (September).

Vos, Rob, J. Cuesta, M. León, R. Lucio y J. Rosero. 2004. "Health". En: World Bank and Inter-American Development Bank (2004) Ecuador: Creating fiscal space for poverty reduction. A fiscal management and public expenditure review, Volume II, Report No. 28911-EC, Washington, D.C.: The World Bank.

Vos, Rob, and Juan Ponce. 2004. "Education." In Ecuador: Creating Fiscal Space for Poverty Reduction, by The World Bank and InterAmerican Development Bank, Volume II:33-66. A Fiscal Management and Public Expenditure Review 28911. The World Bank.

World Health Organization - UNICEF. (2010). Progress in Sanitation and Drinking-Water 2010 .NY: Unicef.

World Health Organization (2003). Global strategy for infant and young child feeding. Geneva: World Health Organization.

World Health Organization (2006). Report of a WHO technical consultation on birth spacing. Geneva: World Health Organization. 